\title{
BMJ Open Effect of skin-to-skin contact on parents' sleep quality, mood, parent-infant interaction and cortisol concentrations in neonatal care units: study protocol of a randomised controlled trial
}

Charlotte Angelhoff, ${ }^{1,2}$ Ylva Thernström Blomqvist, ${ }^{3}$ Charlotte Sahlén Helmer, ${ }^{1,2}$ Emma Olsson, ${ }^{4}$ Shefaly Shorey, ${ }^{5}$ Anneli Frostell, ${ }^{6}$ Evalotte Mörelius ${ }^{1}$

To cite: Angelhoff C, Blomqvist YT, Sahlén Helmer C, et al. Effect of skin-to-skin contact on parents' sleep quality, mood, parent-infant interaction and cortisol concentrations in neonatal care units: study protocol of a randomised controlled trial. BMJ Open 2018;8:e21606. doi:10.1136/ bmjopen-2018-021606

- Prepublication history for this paper is available online. To view these files, please visit the journal online (http://dx.doi. org/10.1136/bmjopen-2018021606).

Received 8 January 2018 Revised 30 April 2018 Accepted 6 July 2018
Check for updates

(C) Author(s) (or their employer(s)) 2018. Re-use permitted under CC BY-NC. No commercial re-use. See rights and permissions. Published by BMJ.

For numbered affiliations see end of article.

Correspondence to Dr Charlotte Angelhoff; charlotte.angelhoff@liu.se

\section{ABSTRACT}

Introduction Separation after preterm birth is a major stressor for infants and parents. Skin-to-skin contact (SSC) is a method of care suitable to use in the neonatal intensive care unit (NICU) to minimise separation between parents and infants. Less separation leads to increased possibilities for parent-infant interaction, provided that the parents' sleep quality is satisfactory. We aimed to evaluate the effect of continuous SSC on sleep quality and mood in parents of preterm infants born $<33$ weeks of gestation as well as the quality of parent-infant interaction and salivary cortisol concentrations at the time of discharge.

Methods and analysis A randomised intervention study with two arms - intervention versus standard care. Data will be collected from 50 families. Eligible families will be randomly allocated to intervention or standard care when transferred from the intensive care room to the family-room in the NICU. The intervention consists of continuous SSC for four consecutive days and nights in the family-room. Data will be collected every day during the intervention and again at the time of discharge from the hospital. Outcome measures comprise activity tracker (Actigraph); validated self-rated questionnaires concerning sleep, mood and bonding; observed scorings of parental sensitivity and emotional availability and salivary cortisol. Data will be analysed with pairwise, repeated measures, Mann Whitney U-test will be used to compare groups and analysis of variance will be used to adjust for different hospitals and parents' gender.

Ethics and dissemination The study is approved by the Regional Research Ethics Board at an appropriate university (2016/89-31). The results will be published in scientific journals. We will also use conferences and social media to disseminate our findings.

Trial registration number NCT03004677.

\section{INTRODUCTION}

Considerable effort has been put into improving the professional care of preterm infants. Nowadays, several care methods aimed at decreasing the amount of stress for the infant (ie, pain relief, fewer procedures
Strengths and limitations of this study

- The intervention will last for four consecutive days starting after at least one nights' sleep together as a family in a single family room, when the infant no longer is in need of intensive care in the intensive care room in the neonatal intensive care unit (NICU).

- Outcomes will be measured by both subjective and objective measurements such as sleep diary, questionnaires, actigraphy, observations and saliva for cortisol analysis.

- This study is not designed to evaluate the long-term consequences or benefits of continuous skin-to-skin contact in the NICU.

and increased respect for the infants' sensitivity) are routine in many neonatal intensive care units (NICUs). Despite this, preterm birth is associated with poorer school performance and developmental and cognitive problems. ${ }^{12}$ This could be a consequence of excessive levels of the stress hormone cortisol acting on the prefrontal cortex and the hippocampus region of the brain. ${ }^{3}$ As one of the most potent stressors in early life is separation from the parent, it is important to actively focus on early proactive parental involvement, that is, continuous skin-toskin contact (SSC). Worldwide, we know that there is an underuse of continuous SSC in NICUs, especially when the infant is discharged from the intensive care room to the family room. The nurses' support, knowledge and attitudes affect the parents' choice and opportunities to perform SSC. ${ }^{45}$ NICU nurses, in a recent study, have expressed beliefs that mothers could feel trapped when practicing continuous SSC and that sleeping with the infant during SSC will hinder good 
parental sleep and thus increase the risk of depressive symptoms. ${ }^{4}$

Poor sleep quality affect the parents' mood and quality of care provided to the infant. ${ }^{6}$ Mothers and fathers in the NICU have previously described that they were more able to relax when they cared for their preterm infant with SSC. ${ }^{6}$ On the other hand, fathers in another study found it difficult to eat and sleep with the infant while giving SSC, as they tried to lie absolutely still and semireclining in the same position, to ensure the infant's comfort. ${ }^{7}$ If the parents share the responsibility of SSC for the preterm infant, it might improve their quality of sleep. By encouraging each other to take turns and assist each other with practical support, the opportunities to provide SSC may be facilitated and manifold. ${ }^{58}$

To our best knowledge, the effects of continuous SSC, after transfer to the family-room, on extremely and very preterm infants' hypothalamic-pituitary-adrenal (HPA) axis and the quality of the parent-infant interaction and parental sleep have not been studied previously. The results of this interventional study will provide further information about parents' sleep and mood during continuous SSC and whether a late and relatively short intervention, such as SSC for 4 days in the family-room, can improve parent-infant interaction and salivary cortisol coregulation.

\section{BACKGROUND}

\section{Being a parent of a preterm infant}

Every year around 15 million infants worldwide are born preterm. Being a parent of a preterm infant affects parenthood in a more negative way than being a parent to a healthy, full-term infant. Parents of preterm infants are exposed to more stressors related to the infants' health condition and need of intensive care than parents of fullterm infants. ${ }^{9}$ Therefore, parents of preterm infants are at greater risk of postpartum depression, post-traumatic stress disorder and other mood swings that affect the quality of parent-infant interaction. ${ }^{10-13}$ Anxiety due to the preterm infants' medical conditions can result in less interaction and increased distance between the parents and infant. ${ }^{10}$ Mothers of preterm infants who are experiencing stress while the infants are in hospital tend to be less responsive towards their infants' communicative signals. ${ }^{12}$ Parents with preterm infants often feel powerless and incapable of providing the care their infants need because they are separated from their infant. This may lead to inactive parenting that can negatively affect the parent-infant interaction. ${ }^{11}$

\section{Parent-infant interaction}

Loss of parental proximity and stimulation are two of the most potent stressors in early life. Infants experiencing long-standing early life stress such as deprivation or neglect show atypical patterns of diurnal HPA activity, with lower morning cortisol levels. A reorganisation of the stress response system (the HPA-axis) requires that the infant experience safeness, that is, a relationship with a consistently sensitive and responsive caregiver. ${ }^{14}$ Maternal sensitivity, defined as the mother's ability to read and respond in a sensitive and adequate way to their infant's communicative signals, is associated with the development of secure attachment. Sensitive mothers tend to have securely attached infants while less sensitive mothers are more likely to have infants classified as insecurely attached. ${ }^{15}$ Infants with less sensitive mothers have higher levels of cortisol in response to handling than infants with sensitive mothers. ${ }^{16}$ As the mother-to-infant bonding behaviours include touch, skin contact, eye-to-eye contact and soothing, it is important that mother and infant are close together after delivery to strengthen this bonding. ${ }^{17}$ However, extremely and very preterm infants are often transferred to intensive care immediately after birth. The infants are placed in an incubator, which decrease the parents' possibility to bond with their infant.

Preterm infants are, owing to their immature neurological system, less capable than full-term infants in showing their needs through communicative signals. Consequently, parents of preterm infants have more difficulties reading and responding accurately to their infant. ${ }^{18}$ The NICU environment in itself (eg, with incubators and ventilators) creates a barrier to parenting, ${ }^{19}$ which may negatively influence parent-infant interaction and thus the infant's long-term cognitive and physical developmental outcome.

\section{Skin-to-skin contact}

SSC is a method of care aiming to minimise separation of mothers and infants. ${ }^{20}$ In SSC, parents carry the infant in an upright position placed skin-to-skin on the chest. Today SSC is used, more or less, all over the world, even for extremely preterm infants. ${ }^{21}$ In the beginning, infants are mostly taken out from their incubators a few hours per day to be cared for in intermittent SSC with the parents. Along with the infant's maturation, the time spent in SSC/ day increases successively and eventually parents may care for their preterm infant via continuous SSC (ie, around the clock). Continuous SSC has so far been insufficiently evaluated in level III NICUs. ${ }^{20}$ One earlier study performed in Sweden found that the median daily duration of SSC was $<7$ hours for infants born at GA $28+0$ to $33+6 .{ }^{22}$ There are no studies investigating the effects of an intervention comprising continuous SSC with extremely and very preterm infants after the transfer to a family-room (level II NICU) from the intensive care room (level III NICU).

\section{Benefits of SSC}

SSC is beneficial for preterm infants, as it contributes to cardiorespiratory stabilisation, fewer signs of stress and a more organised sleep-wake cycle. ${ }^{23-25}$ In mothers, SSC improves mood, reduces stress and postpartum depression symptoms, encourages them to create a stimulating and care giving environment and promotes breastfeeding. ${ }^{23}{ }_{26-29}$ Few studies of SSC include fathers. ${ }^{30}$ Yet, fathers participating in SSC have rated lesser spousal 
relationship problems when their preterm infants were 4 months corrected age than fathers who did not practice SSC. ${ }^{31}$ Fathers' opportunities to be a part of the SSC may improve their paternal role $^{7}$ and help them transition into a more equal parenthood. ${ }^{32}$

\section{Sleep quality}

Sleep quality is subjective and described as the duration of sleep followed by spontaneous awakening and alertness throughout the day. ${ }^{33}$ Poor sleep quality leads to increased negative emotions that in turn disrupt sleep, which further impairs emotional well-being. ${ }^{34}$ For instance, several awakenings during night hinder deep sleep, which lead to sleepiness and insufficient recovery from a stressful day. ${ }^{35}$ Poor sleep quality may result in sleep-related daytime dysfunctions such as maintaining enthusiasm to complete daily tasks and parental ability as well as motivation to adequately care for their child ${ }^{36}$ and negative moods such as frustration, impatience and irritability against the child. ${ }^{37}$ Worries and uncertainty about the infant's medical situation may also negatively influence parents' sleep quality. In two recent qualitative studies, parents of sick children have described that they avoid falling into deep sleep in case something untoward should happen to the child. ${ }^{638}$

\section{Cortisol}

Stress response is a necessary physiological and biological response aimed at maintaining homeostasis when the individual is exposed to a stressor. Stress reactivity is when a stressor triggers the HPA-axis to release the stress hormone cortisol into the blood stream. ${ }^{39}$ When there is adequate cortisol to handle the situation, the levels decline to baseline (recovery). Cortisol is also released in a diurnal circadian rhythm with the highest levels in the morning, a steady decrease throughout the day and the lowest levels at night. The circadian rhythm develops during the infants' first year of life. ${ }^{40}{ }^{41}$ It has been suggested that genetic as well as environmental factors can affect the development of the cortisol circadian rhythm and coregulation between the mother's and infant's cortisol levels. For instance, a coregulation in salivary cortisol levels has been shown between preterm infants and their parents at discharge from the NICU if the parent had slept in the NICU compared with when the parent slept at home ${ }^{42}$ and between 3-month-old full-term infants and their parents prior to vaccination. ${ }^{43} \mathrm{~A}$ coregulation in salivary cortisol levels has also been shown between 6 -month-old full-term infants and their mothers, but not the fathers. This was explained by the fact that infants spent most of their time in the same environment as their mothers, who were the primary caregivers. ${ }^{44}$ Moreover, a coregulation in salivary cortisol levels has been described for twins sharing the same environment but not for twins living in different environments. ${ }^{42}{ }^{45}$ For mothers practicing continuous SSC with their preterm infant in the NICU, a coregulation in salivary cortisol was shown between the mother and the infant at 4 months corrected age but not in the control group. ${ }^{32}$

\section{Aim}

The aim of the study is to evaluate the effect of continuous SSC on sleep quality and mood in parents of preterm infants born $<33$ weeks of gestation as well as parent-infant interaction and salivary cortisol concentrations at the time of discharge.

Our hypothesis is that SSC for four consecutive days during the first time they are given the opportunity to sleep together as a family will function as a restart for bonding and therefore improve the parents' mood, interaction behaviour and coregulation of salivary cortisol between parent and infant. Furthermore, our hypothesis is that the parents' possibility to take turns and assist each other with practical support during SSC will facilitate parents' sleep quality.

\section{Research questions}

Is there a statistically significant difference in the effect of continuous SSC compared with standard care on the parents' sleep quality and mood?

Is there a statistically significant difference in the effect of continuous SSC compared with standard care on the quality of interaction between the parent and preterm infant at the time of discharge?

Is there a statistically significant difference in the effect of continuous SSC compared with standard care on salivary cortisol reactivity, recovery and coregulation between parents and preterm infants at the time of discharge?

\section{METHODS AND ANALYSIS \\ Design}

This study is a randomised controlled trial.

\section{Statistics}

Calculation of power indicates that 48 parents are needed in each group for an effect size of $0.7(=0.01 ;=0.80)$ in the variable sleep quality. Because of the risk of dropout, the goal is to include 25 families (50 parents) in each group. Data will be analysed within the family dyads as well as individually to control for lack of independence. Sleep variables and mood will be analysed with pairwise, repeated measures. Parent-infant interaction will be analysed with descriptive statistics. Saliva cortisol will be analysed with pairwise, repeated measures and correlation analyses. Mann Whitney U-test will be used to compare groups and analysis of variance will be used to adjust for different hospitals, parents' gender and time in SSC. The statistical software program SPSS will be used for analyses.

\section{Intervention}

Parents will receive written and oral information about the study by a research coordinator before randomisation. The families who accept participation will be randomised to receive either continuous SSC (intervention group=IG) or standard care (control group $=\mathrm{CG}$ ). Sequentially numbered envelopes generated by a statistical randomisation programme operated by the first author will be 


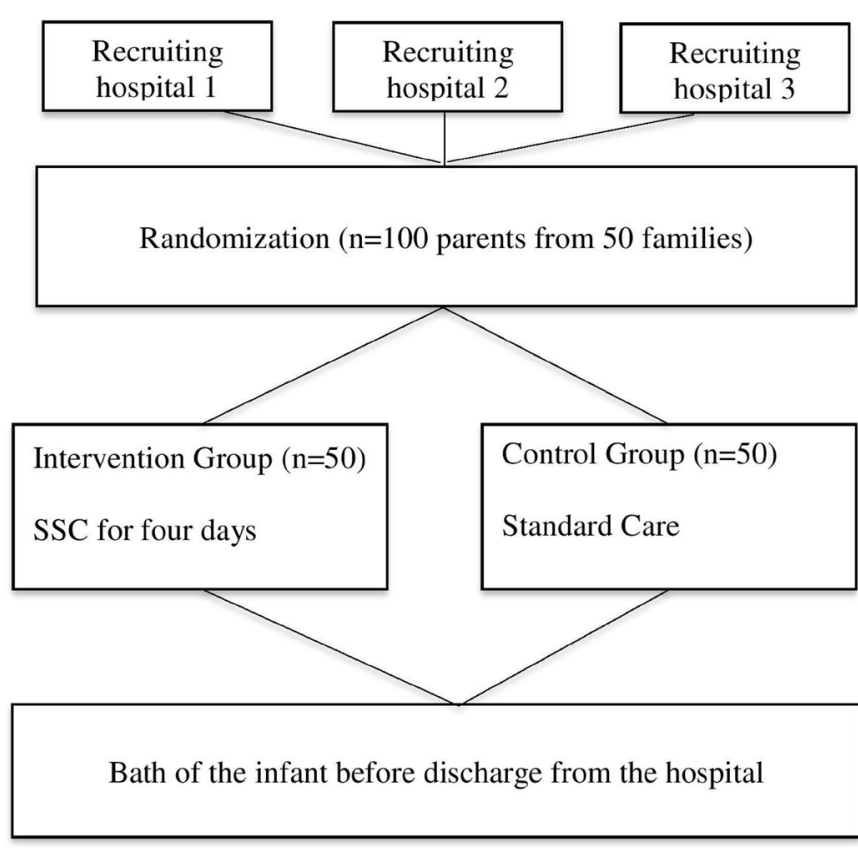

Figure 1 The CONSORT diagram of this study. SSC, skinto-skin contact.

used for randomisation. The randomisation sequence is described in a Consort flowchart (figure 1). The intervention will last for four consecutive days starting after at least one nights' sleep together as a family in a single family room, when the infant no longer is in need of intensive care in the intensive care room in the NICU. Four days were chosen to study if a short intervention is feasible to affect parents' sleep, mood and parent-infant interaction, but also to avoid the risk of overload the families and of dropouts due to early discharge. In families assigned to the IG, the infants will be cared for by SSC on parents' chest 24 hours a day, alternating between the parents. The parents will note who provides the SSC, and if and for how long they are off SSC for any reason.

Families randomised to CG are free to practice intermittent SSC as much as they want. The parents will note with whom, when and for how long they provide SSC.

In the present study, standard care (CG) is defined as NICU care when the infant stay with its parents around the clock and is cared for skin-to-skin with its parents as much as the parents wish. NICU care for the infants in IG and CG are the same, but may partly differ between the NICUs. Because of ethical reasons, we cannot refrain the parents from using SSC. The only thing that separates the groups is the time the infant is cared for SSC during the four study days.

\section{Settings}

Family-centred NICUs (level II-III), in three university hospitals and two general hospitals in Sweden, with single family rooms where the family stay together with their infant around the clock when intensive care is no longer required.

The parents' presence at NICUs in Sweden are facilitated through the Swedish national insurance system, which allows both parents to share parental benefit for 480 days per child. In addition, both parents of an infant requiring care at a NICU are entitled to 'temporary parental benefit' until the infant is discharged. This means that during the infant's entire NICU stay, both parents have the legal right to be together with their infant at the NICU. Further, in Sweden, hospital care for all children is free of charge.

\section{Inclusion criteria}

Parents (mother and father/partner) of single preterm infants born $<33$ weeks of gestation will be included. The infant should have moved out from the intensive care room and into a single family room where both parents can stay and care for their infant 24 hours a day. Parents need to be able to read and understand the questionnaires. Exclusion criteria are parents with sleeping disorders, psychiatric problems or drug abuse and infants with major congenital malformation, grade III-IV intraventricular haemorrhage or a chromosomal defect that could affect the infant's ability to interact.

How old the infants should be, as well as infant criteria, to be cared for in a single family room varies between the NICUs. Most often, this happens as soon as the infant no longer need ventilator therapy (except for oxygen cannula). The time the infants are cared for in a family room before discharge varies greatly, partly depending on the infant's and parent's well-being, the NICUs routines and access to further care at home.

\section{Procedure}

Figure 2 shows the study sequence. A pilot test has been performed to test the feasibility of the study in two families. Because of the parents' experience of the time it took to complete the questionnaires, minor changes were made. Two questionnaires (Sense of coherence and SF-36) were removed from the study. Moreover, data collection in the evening (Mood Adjective Checklist, MACL) was removed during the intervention.

\section{Outcome measurements}

\section{Parents' sleep quality}

The primary outcome of this study is sleep quality. A study-specific sleep diary for four consecutive days will be used to provide data on perceived sleep quality on a five-point scale ( $1=\mathrm{bad}, 5=$ good). The diary includes the variables time going to bed, sleep onset latency, morning awakening time, sleep duration, nocturnal awakenings, daytime napping and sleep efficiency. The diary also consists of open-ended questions about potential variables affecting sleep.

A validated actigraph-Actigraph Link GT9X activity monitor-will be used to measure physical activity and sleep continuously during the 4-day intervention period. The actigraph will be placed around the waist to suit hygiene recommendations in the NICU.

\section{Parents' mood}

Parents will fill out the MACL at baseline, in the mornings during the intervention and at discharge. The MACL is 


\begin{tabular}{|c|c|c|c|c|}
\hline \multicolumn{4}{|c|}{ Primary outcome } & Secondary outcome \\
\hline \multicolumn{2}{|c|}{$\begin{array}{l}\text { Informed written and verbal consent } \\
\text { Randomisation }\end{array}$} & $\begin{array}{l}\text { Baseline/Pre-test } \\
\text { Day 1, 8.00 PM }\end{array}$ & $\begin{array}{c}\text { Intervention Day } \\
2-5,8.00 \mathrm{AM}\end{array}$ & $\begin{array}{l}\text { Post-test At } \\
\text { discharge }\end{array}$ \\
\hline $\begin{array}{l}\text { Intervention group } \\
\text { (IG) }\end{array}$ & Control group (CG) & $I G+C G$ & $I G+C G$ & \multirow{2}{*}{$\begin{array}{l}\text { Uppsala Sleep Inventory (USI) } \\
\text { Mother to Infant Bonding Scale } \\
\text { (MIBS) } \\
\text { The Edinburgh Postnatal } \\
\text { Depression Scale (EPDS) } \\
\text { State Trait Anxiety Inventory } \\
\text { (STAI) } \\
\text { The parents will be performing a } \\
\text { bath of the infant, which will be } \\
\text { filmed and analysed later } \\
\text { (Emotional Availability and } \\
\text { Ainsworth Sensitivity Scale). } \\
\text { Saliva cortisol samples from botr } \\
\text { parents and the infant before a } \\
\text { bath and } 30 \text { min after } \\
\text { the beginning of the bath }\end{array}$} \\
\hline $\begin{array}{l}\text { Parents will provide } \\
\text { continuous skin to skin- } \\
\text { care } 24 \text { hours a day } \\
\text { alternating between the } \\
\text { parents with start } 8.00 \\
\text { pm, day } 1 \\
\text { Parents will be provided } \\
\text { with clothes, i.e. tube } \\
\text { tops, scarves, and } \\
\text { blouses to facilitate the } \\
\text { care }\end{array}$ & & $\begin{array}{l}\text { Activity tracker } \\
\text { Skin to skin-log }\end{array}$ & & \\
\hline
\end{tabular}

Figure 2 Study schedule.

a self-administered, validated instrument measuring the bipolar dimensions of mood. The instrument consists of 71 adjectives (eg, 'secure', 'relaxed', 'attached', 'happy', 'energetic' and 'sociable') measured on a four-point scale (1=it definitely disagrees with what I feel right now, $4=$ it definitely agrees with what I feel right now), divided into six dimensions: control, calmness, social orientation, pleasantness, activation and extraversion. The instrument refers to how the responder feels at the moment. ${ }^{47}$

Parents will fill out Spielberger's State Trait Anxiety Inventory (STAI) at baseline and at discharge. The STAI is a self-administrated questionnaire measuring state and trait anxiety. The short version of STAI is a valid and reliable questionnaire consisting of six statements measured on a four-point scale. The questionnaire refers to how the responder feels at the moment. ${ }^{48}$

Parents will fill out Edinburgh Postnatal Depression Scale (EPDS) at baseline and at discharge. The EPDS is a validated, self-administrated questionnaire developed to discover depression in new mothers. The scale consists of 10 statements measured on a four-point scale. The scale refers to how the responder has felt in the last 7 days. ${ }^{50}$

Parent-infant interaction

Parent-infant interaction will be measured at time for discharge using the Mother-Infant Bonding Scale (MIBS), Emotional availability (EA) scale and Ainsworth's sensitivity scale.
The MIBS is a reliability tested, self-rating mother-to-infant bonding questionnaire designed to assess the early feelings of a mother towards her infant. The MIBS includes eight items measured on a four-point scale. The scale refers to how the responder has felt in the last 7 days. $^{51}$

The EA scale measures the parent-infant relationship. Five scales focus on the parent and two scales on the infant. ${ }^{52}$ A certified objective rater assesses the parent-infant dyad from a video recording of an interplay activity, in this study this is a bath of the infant, performed by the parents.

Ainsworth's sensitivity scale consists of four nine-point scales, with five anchor points on each scale, measuring sensitivity, cooperation, availability and acceptance. ${ }^{15} \mathrm{~A}$ trained objective rater assesses the parent-infant dyad from a video recording of an interplay activity (bath).

\section{Salivary cortisol reactivity, recovery and coregulation}

Saliva for cortisol analysis will be collected from both parents and the infant before (baseline) and 30 (reactivity) and 40 (recovery) min after starting a bath for the infant (stressor). ${ }^{53}$ The coregulation will be calculated as a paired correlation between the parents' and infant's cortisol levels. Saliva will be collected with Salimetrics oral swabs (Salimetrics, Pennsylvania, USA). The saliva samples will be centrifuged and stored at $-80 \mathrm{C}$, until further analysis at the University hospital. ${ }^{54-56}$ 


\section{Measurements of possible confounding factors}

Sleep quality

Thirteen items will be used from The Uppsala Sleep Inventory, measuring perceived sleep quality and consequences of sleep loss. The questionnaire evaluates perceived sleep retrospectively referring to the past 4 weeks. In this study, it will reflect the time since the infant was born. The questionnaire has been validated against polysomnography and actigraphy and is reliability tested. ${ }^{57}$

\section{Background data}

Demographic and medical data, for infants and parents, will be collected to include age; education; marital and health status; information about birth (weight, APGAR score, birth condition); breastfeeding and the time in SSC and/or other activities performed in the NICU during the intervention period.

\section{Stressor}

The parents will give the infant a bath on the day of, or before, hospital discharge. The bath situation will be video recorded with a handy camera for later interpretation of parent-infant interaction.

\section{Patient and public involvement}

The development of research questions is based on previous qualitative studies describing patients' and NICU staff's experiences and preferences. The families have not been involved in the design and will not be involved in recruitment or conduct of the study. The results will be disseminated to study participants through media and information at the NICU wards.

\section{ETHICS AND DISSEMINATION}

The study is registered at ClinicalTrials.gov (NCT03004677).

The parents will receive oral and written information about the study before they give written, informed consent. They will be informed that they can decline to participate or can withdraw from the study at any time without explanation or consequences for their infant's future care. All collected data will be coded and are stored in separate, secure locations. Saliva samples will be collected and stored according to the Swedish law SFS 2002:297 on biobanks in healthcare with approval from the Regional Biobank Center. All saliva samples will be destroyed after analysis. The primary investigator is responsible for communicating important protocol modifications to relevant parties.

There is no physical or psychological harm related to the study. Families randomised to the CG are welcome to care for their infant in SSC for as much as they want. The amount of SSC these infants experience is recorded and when necessary, controlled for in the statistical analyses. The interpretation of the results will be adjusted accordingly.
Salivary cortisol sampling is painless and does not include any risks. As questionnaires can violate personal integrity, all questionnaires to be used in this study are well established, and participation in the study is voluntary and can be withdrawn at any time. The stressor used in this study, that is, bath, is performed by the parents and is part of the infants' normal care.

Normally, extremely and very preterm infants face the greatest risk of impaired development. Considering that 15 million infants are born preterm every year, it makes a great impact on the society at large. The risk of impaired cognitive development is further affected by high levels of cortisol and the quality of parent-infant interaction. For the individual infant that was born preterm, an improved cognitive development is certainly a tremendous gain, providing a healthier life with less cognitive disabilities and associated consequences. Each child that gains a healthier cognitive development also has great economic impact on the societal level, including reduced need for medical care during the entire childhood because of (1) less cognitive impairments such as visual and hearing problems, attention disorders and motor difficulties as well as (2) less psychosocial ill health such as socioemotional problems, aggressions and depressions. An improved cognitive and socioemotional functioning, with less concentration and learning difficulties is also a great gain when it comes to school achievement and working life. As the bonding process and parent-infant interaction start right after birth, they are both affected negatively by parent-infant separation. If the transfer to family-room, including continuous SSC, can be used to rekindle the bonding process, it will facilitate parent-infant interaction. Moreover, if the NICU staff can support the family by providing methods with potential to hinder separation, it may have a tremendous impact on the individual child's life, family and society. Parker and Anderson have described positive outcomes of SSC in adoptive parents and suggest that biological parents of a critically ill preterm infant feel somewhat like adoptive parents after the prolonged period of time in NICU, customarily unable to hold their infant. ${ }^{58}$ Moreover, SSC seems to work as a restart of breastfeeding in full-term infants after having latch-on problems. ${ }^{59}$ In order to provide evidence-based methods, continuous SSC needs to be evaluated concerning parents' sleep quality, because poor sleep quality may affect parents' mood and the ability to interact with, and care for, the fragile preterm infant.

The results of this study will be published in scientific journals, international and national conferences.

\section{Author affiliations}

${ }^{1}$ Department of Social and Welfare Studies, Division of Nursing Science, Linköping University, Norrköping, Sweden

${ }^{2}$ Department of Clinical and Experimental Medicine and Department of Paediatrics, Linköping University, Linköping, Sweden

${ }^{3}$ Department of Women's and Children's Health, Uppsala University, Uppsala, Sweden

${ }^{4}$ Department of Pediatrics and Centre for Health Care Sciences, Örebro University, Örebro, Sweden 
${ }^{5}$ Alice Lee Centre for Nursing Studies, Yong Loo Lin School of Medicine, National University of Singapore, National University Health System, Singapore, Singapore ${ }^{6}$ Division of Psychology, Department of Behavioral Sciences and Learning, Linköping University, Linköping, Sweden

Acknowledgements The authors' would like to thank US Foundation for Medical Research (Sweden), Ebba Danelius Foundation (The Swedish Society of Nursing) and Första Majblomman Research Foundation i Östergötland (Sweden) for financial support.

Contributors All authors contributed to the study design. CA and EM had primary responsibility of writing the study protocol. YTB, CSH, EO, SS and AF critically revised the protocol.

Funding This work is funded by County Council of Östergötland (LIO-720151 and LI0-663781) and The Medical Research of Southeast Sweden (FORSS-661721).

Competing interests None declared.

Patient consent Not required.

Ethics approval The study has been approved by the Regional Research Ethics Board at an appropriate university (2016/89-31).

Provenance and peer review Not commissioned; externally peer reviewed.

Open access This is an open access article distributed in accordance with the Creative Commons Attribution Non Commercial (CC BY-NC 4.0) license, which permits others to distribute, remix, adapt, build upon this work non-commercially, and license their derivative works on different terms, provided the original work is properly cited, appropriate credit is given, any changes made indicated, and the use is non-commercial. See: http://creativecommons.org/licenses/by-nc/4.0/.

\section{REFERENCES}

1. Jarjour IT. Neurodevelopmental outcome after extreme prematurity: a review of the literature. Pediatr Neurol 2015;52:143-52.

2. Baron IS, Litman FR, Ahronovich MD, et al. Late preterm birth: a review of medical and neuropsychological childhood outcomes. Neuropsychol Rev 2012;22:438-50.

3. Lombroso PJ, Sapolsky R. Development of the cerebral cortex: XII. Stress and brain development: I. J Am Acad Child Adolesc Psychiatry 1998;37:1337-9.

4. Mörelius E, Anderson GC. Neonatal nurses' beliefs about almost continuous parent-infant skin-to-skin contact in neonatal intensive care. J Clin Nurs 2015;24:2620-7.

5. Blomqvist YT, Frölund L, Rubertsson C, et al. Provision of Kangaroo Mother Care: supportive factors and barriers perceived by parents. Scand J Caring Sci 2013;27:345-53.

6. Edéll-Gustafsson U, Angelhoff C, Johnsson E, et al. Hindering and buffering factors for parental sleep in neonatal care. A phenomenographic study. J Clin Nurs 2015;24:717-27.

7. Blomqvist YT, Rubertsson C, Kylberg E, et al. Kangaroo Mother Care helps fathers of preterm infants gain confidence in the paternal role. J Adv Nurs 2012;68:1988-96.

8. Blomqvist YT, Nyqvist KH, Rubertsson C, et al. Parents need support to find ways to optimise their own sleep without seeing their preterm infant's sleeping patterns as a problem. Acta Paediatr 2017; $106: 223-8$

9. Evans T, Whittingham K, Sanders M, et al. Are parenting interventions effective in improving the relationship between mothers and their preterm infants? Infant Behav Dev 2014;37:131-54.

10. Hall RA, Hoffenkamp HN, Tooten A, et al. Longitudinal associations between maternal disrupted representations, maternal interactive behavior and infant attachment: a comparison between full-term and preterm dyads. Child Psychiatry Hum Dev 2015;46:320-31.

11. Jiang S, Warre R, Qiu X, et al. Parents as practitioners in preterm care. Early Hum Dev 2014;90:781-5.

12. Borghini A, Habersaat S, Forcada-Guex M, et al. Effects of an early intervention on maternal post-traumatic stress symptoms and the quality of mother-infant interaction: the case of preterm birth. Infant Behav Dev 2014;37:624-31.

13. Vigod SN, Villegas L, Dennis CL, et al. Prevalence and risk factors for postpartum depression among women with preterm and low-birthweight infants: a systematic review. BJOG 2010;117:540-50.

14. Loman MM, Gunnar MR; Early Experience, Stress, and Neurobehavioral Development Center. Early experience and the development of stress reactivity and regulation in children. Neurosci Biobehav Rev 2010;34:867-76.
15. Ainsworth MDS, Bell SM, Stayton DF. Infant-mother attachment and social development: Socialization as a product of reciprocal responsiveness to signals. Richards \& Richards (eds). New York: Cambridge University Press, 1974.

16. Mörelius E, Nelson N, Gustafsson PA. Salivary cortisol response in mother-infant dyads at high psychosocial risk. Child Care Health Dev 2007;33:128-36.

17. Klaus $\mathrm{MH}$, Jerauld R, Kreger NC, et al. Maternal attachment. Importance of the first post-partum days. $N$ Engl J Med 1972;286:460-3.

18. Clark CA, Woodward LJ, Horwood LJ, et al. Development of emotional and behavioral regulation in children born extremely preterm and very preterm: biological and social influences. Child Dev 2008;79:1444-62.

19. Aagaard H, Hall EOC. International Pediatric Nursing: Mothers' Experiences of Having a Preterm Infant in the Neonatal Care Unit: A Meta-Synthesis. J Pediatr Nurs 2008;23:e26-36.

20. Conde-Agudelo A, Díaz-Rossello JL. Kangaroo mother care to reduce morbidity and mortality in low birthweight infants. Cochrane Database Syst Rev 2016:CD002771.

21. Blomqvist YT, Ewald U, Gradin M, et al. Initiation and extent of skin-to-skin care at two Swedish neonatal intensive care units. Acta Paediatr 2013;102:22-8.

22. Mörelius $\mathrm{E}$, Angelhoff $\mathrm{C}$, Eriksson J, et al. Time of initiation of skin-toskin contact in extremely preterm infants in Sweden. Acta Paediatr 2012;101:14-18.

23. Mörelius E, Theodorsson E, Nelson N. Salivary cortisol and mood and pain profiles during skin-to-skin care for an unselected group of mothers and infants in neonatal intensive care. Pediatrics 2005;116:1105-13.

24. McCain GC, Ludington-Hoe SM, Swinth JY, et al. Heart rate variability responses of a preterm infant to kangaroo care. J Obstet Gynecol Neonatal Nurs 2005;34:689-94.

25. Ludington-Hoe SM, Johnson MW, Morgan K, et al. Neurophysiologic assessment of neonatal sleep organization: preliminary results of a randomized, controlled trial of skin contact with preterm infants. Pediatrics 2006;117:e909-e923.

26. Gathwala G, Singh B, Balhara B. KMC facilitates mother baby attachment in low birth weight infants. Indian J Pediatr 2008;75:43-7.

27. Neu M, Hazel NA, Robinson J, et al. Effect of holding on coregulation in preterm infants: a randomized controlled trial. Early Hum Dev 2014;90:141-7.

28. Neu M, Laudenslager ML, Robinson J. Coregulation in salivary cortisol during maternal holding of premature infants. Biol Res Nurs 2009;10:226-40.

29. Renfrew MJ, Dyson L, McCormick F, et al. Breastfeeding promotion for infants in neonatal units: a systematic review. Child Care Health Dev 2010;36:165-78.

30. Shorey S, He H-G, Morelius E. Skin-to-skin contact by fathers and the impact on infant and paternal outcomes: an integrative review. Midwifery 2016;40:207-17.

31. Mörelius E, Örtenstrand A, Theodorsson E, et al. A randomised trial of continuous skin-to-skin contact after preterm birth and the effects on salivary cortisol, parental stress, depression, and breastfeeding. Early Hum Dev 2015;91:63-70.

32. Olsson E, Eriksson M, Anderzén-Carlsson A. Skin-to-Skin Contact Facilitates More Equal Parenthood - A Qualitative Study From Fathers' Perspective. J Pediatr Nurs 2017;34:e2-e9.

33. Porkka-Heiskanen T, Zitting KM, Wigren HK. Sleep, its regulation and possible mechanisms of sleep disturbances. Acta Physiol 2013;208:311-28.

34. Kahn M, Sheppes G, Sadeh A. Sleep and emotions: bidirectional links and underlying mechanisms. Int J Psychophysiol 2013;89:218-28.

35. Markov D, Goldman M. Normal sleep and circadian rhythms: neurobiologic mechanisms underlying sleep and wakefulness. Psychiatr Clin North Am 2006;29:841-53.

36. Herbert LJ, Monaghan M, Cogen F, et al. The impact of parents sleep quality and hypoglycemia worry on diabetes self-efficacy. Behav Sleep Med 2015;13:308-23.

37. Cooklin AR, Giallo R, Rose N. Parental fatigue and parenting practices during early childhood: an Australian community survey. Child Care Health Dev 2012;38:654-64.

38. Angelhoff C, Edéll-Gustafsson U, Mörelius E. Sleep of Parents Living With a Child Receiving Hospital-Based Home Care: A Phenomenographical Study. Nurs Res 2015;64:372-80.

39. McEwen BS. Central effects of stress hormones in health and disease: Understanding the protective and damaging effects of stress and stress mediators. Eur J Pharmacol 2008;583:174-85. 
40. Ivars K, Nelson N, Theodorsson A, et al. Development of Salivary Cortisol Circadian Rhythm and Reference Intervals in Full-Term Infants. PLoS One 2015;10:e0129502.

41. Ivars K, Nelson N, Theodorsson A, et al. Development of salivary cortisol circadian rhythm in preterm infants. PLoS One 2017;12:e0182685.

42. Mörelius E, Broström EB, Westrup B, et al. The Stockholm Neonatal Family-Centered Care Study: effects on salivary cortisol in infants and their mothers. Early Hum Dev 2012;88:575-81.

43. Mörelius E, Theodorsson E, Nelson N. Stress at three-month immunization: parents' and infants' salivary cortisol response in relation to the use of pacifier and oral glucose. Eur J Pain 2009;13:202-8.

44. Stenius F, Theorell T, Lilja G, et al. Comparisons between salivary cortisol levels in six-months-olds and their parents. Psychoneuroendocrinology 2008;33:352-9.

45. Custodio RJ, Junior CE, Milani SL, et al. The emergence of the cortisol circadian rhythm in monozygotic and dizygotic twin infants: the twin-pair synchrony. Clin Endocrinol 2007;66:192-7.

46. Schreiber JE, Shirtcliff E, Van Hulle C, et al. Environmental influences on family similarity in afternoon cortisol levels: twin and parent-offspring designs. Psychoneuroendocrinology 2006;31:1131-7.

47. Sjöberg L, Svensson E, Persson LO. The measurement of mood. Scand J Psychol 1979;20:1-18.

48. Marteau TM, Bekker $\mathrm{H}$. The development of a six-item short-form of the state scale of the Spielberger State-Trait Anxiety Inventory (STAI). Br J Clin Psychol 1992;31:301-6.

49. Schalling D, Cronholm B, Asberg M, et al. Ratings of psychic and somatic anxiety indicants. Interrater reliability and relations to personality variables. Acta Psychiatr Scand 1973;49:353-68.
50. Cox JL, Holden JM, Sagovsky R. Detection of postnatal depression. Development of the 10-item Edinburgh Postnatal Depression Scale. Br J Psychiatry 1987;150:782-6.

51. Taylor A, Atkins R, Kumar R, et al. A new Mother-to-Infant Bonding Scale: links with early maternal mood. Arch Womens Ment Health 2005;8:45-51.

52. Biringen Z, Derscheid D, Vliegen N, et al. Emotional availability (EA): Theoretical background, empirical research using the EA Scales, and clinical applications. Developmental Review 2014;34:114-67.

53. Mörelius E, He HG, Shorey S. Salivary Cortisol Reactivity in Preterm Infants in Neonatal Intensive Care: An Integrative Review. Int J Environ Res Public Health 2016;13:337

54. Morelius E, Nelson N, Theodorsson E. Salivary cortisol and administration of concentrated oral glucose in newborn infants: improved detection limit and smaller sample volumes without glucose interference. Scand J Clin Lab Invest 2004;64:113-8.

55. Mörelius E, Nelson N, Theodorsson E. Saliva collection using cotton buds with wooden sticks: a note of caution. Scand J Clin Lab Invest 2006;66:15-18.

56. Mörelius E, Theodorsson E, Nelson N. Sample volume matters when sampling saliva in paediatric clinical analysis. Acta Paediatr 2013;102:e389.

57. Edéll-Gustaffson UM. Insufficient sleep, cognitive anxiety and health transition in men with coronary artery disease: a self-report and polysomnographic study. J Adv Nurs 2002;37:414-22.

58. Parker L, Anderson GC. Kangaroo care for adoptive parents and their critically ill preterm infant. MCN Am J Matern Child Nurs 2002;27:230-2

59. Svensson KE, Velandia MI, Matthiesen AS, et al. Effects of motherinfant skin-to-skin contact on severe latch-on problems in older infants: a randomized trial. Int Breastfeed J 2013;8:1. 